\title{
Predictive role of telomerase activity in the clinical outcome of patients with benign lesions of the uterine cervix or CIN
}

\author{
Sara Bravaccini ${ }^{\mathrm{a}, *}$, Andrea Amadori ${ }^{\mathrm{b}}$, Emanuela Scarpi $^{\mathrm{a}}$, Maria Maddalena Tumedei ${ }^{\mathrm{a}}$, \\ Wainer Zoli ${ }^{\mathrm{a}}$ and Rosella Silvestrini ${ }^{\mathrm{a}}$ \\ ${ }^{\mathrm{a}}$ IRCCS Istituto Scientifico Romagnolo per lo Studio e la Cura dei Tumori (IRST), Meldola, Italy \\ ${ }^{\mathrm{b}}$ Department of Obstetrics and Gynecology, Morgagni-Pierantoni Hospital, Forlì, Italy
}

Received: March 2, 2012

Accepted: April 25, 2012

\begin{abstract}
Telomerase, a fundamental marker of neoplastic transformation, is widely expressed in both premalignant intraepithelial lesions and in most malignant lesions of the uterine cervix. We determined telomerase activity (TA) in uterine cervix by Repeat Amplification Protocol (TRAP) in a series of 62 cases, 44 with benign diseases (inflammation and/or metaplasia and/or acanthosis) and 18 with cervical intraepithelial neoplasia (CIN). No significant differences in TA were observed between benign lesions (median AEU value 36, range 0-119) and CIN (median AEU value 30, range 0-65). Conversely, TA was significantly higher in subjects who showed CIN evolution (65 range 45-119) than in disease-free individuals (34 range 0-95, $\rho=0.017$ ) and in 1 patient with a CIN2 lesion who relapsed after 5 years. Our results suggest that TA of the uterine cervix is capable of predicting CIN evolution or relapse, thus indicating its potential usefulness as a prognostic marker in clinical surveillance programs.
\end{abstract}

Keywords: Cervical dysplasia, telomerase activity, clinical outcome

\section{Introduction}

The diagnosis of cervical dysplasia and carcinoma is based on the objective assessment of well-defined microscopic features and on the more subjective evaluation of often subtle parameters [2]. However, morphologic evaluation by routine microscopy is not capable of discriminating between cervical lesions that will recur and those that will not (Figs. 1,2). Telomerase, a fundamental marker of neoplastic transformation, is widely expressed in both preneoplastic intraepithelial lesions and in the majority of malignant lesions of the uterine cervix and is therefore a potential

\footnotetext{
${ }^{*}$ Corresponding author: Sara Bravaccini, Biosciences Laboratory, IRCCS Istituto Scientifico Romagnolo per lo Studio e la Cura dei Tumori (IRST), Via P. Maroncelli 40, 47014 Meldola, FC, Italy. Tel.: +39 0543 739226; Fax: +39 0543 739221; E-mail: s.bravaccini@irst.emr.it.
}

candidate for this purpose [7]. Although the diagnostic role of TA has been extensively investigated in several tumor types including cervical cancer [1, 5, 8, 9], its predictive relevance has yet to be defined. In the present study, we retrospectively analyzed TA during different phases of epithelial transformation in the uterine cervix in a preliminary series of patients with benign disease or CIN.

\section{Materials and methods}

Cervical scrapings were collected from a consecutive series of 62 women presenting for cervical screening. A part of the material was sent to the Pathology Laboratory for cytologic evaluation and the remainder was placed in test tubes containing phosphate buffered saline (PBS) and sent to the Molecular Laboratory for 


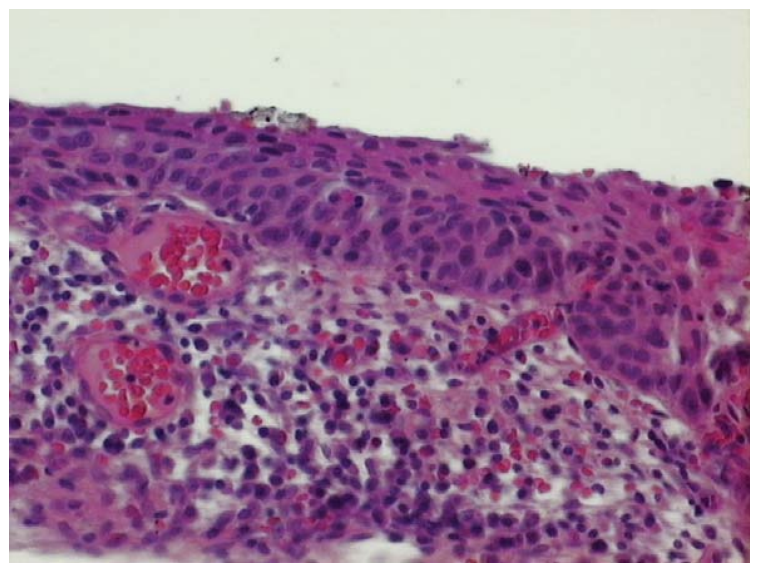

Fig. 1. CIN 3 non-recurring lesion.

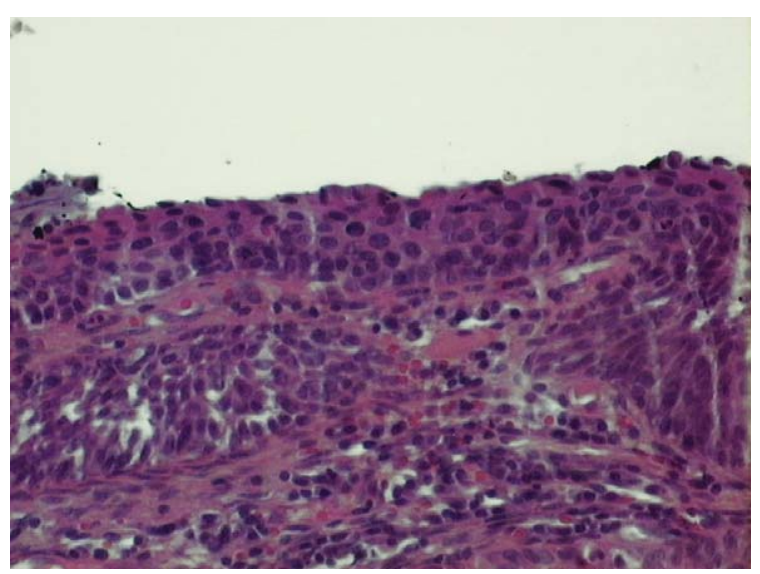

Fig. 2. CIN 3 recurring lesion.

assessment with telomeric repeat amplification protocol (TRAP) assay. Cytologic features were classified according to the 2001 Bethesda system [4].

\subsection{TRAP}

We used the method previously described [3,10], opportunely modified [6]. Specifically, telomerase products were evaluated on fluorescent electropherograms and the area underlying the different peaks was calculated. An internal telomerase assay standard (ITAS; 25 attograms) amplified by the same 2 primers used for telomerase activity (TA) determination was included in the TRAP buffer to obtain a semi-quantitative evaluation. Protein concentrations corresponding to $10,30,100,300,1000$ and 3000 cells from a human bladder cancer cell line (MCR) were analyzed in each assay and used as the reference curve. The areas of each sample were also normalized to the 150-base pair ITAS peak to obtain quantitative TA evaluations. The relative TA per cell for each sample was expressed as the percentage of the ratio of TRAP ladder/ ITAS per cell vs. the value of MCR and expressed in arbitrary enzymatic units (AEUs). The TRAP assay was carried out on the entire case series (feasibility $100 \%)$. All determinations were performed blindly in duplicate and, when variations were $>15 \%$ (observed in about $10 \%$ of cases), a third sample was analyzed. TA was expressed as a continuous variable in all analyses. To analyze the relation between TRAP activity and clinical outcome, patients were subdivided into two groups (disease-free for at least 5 years and disease evolution within 5 years of diagnosis) and a nonparametric ranking statistic (median test) was used.

Accuracy of the TRAP assay, considered as a continuous variable, was evaluated by receiver operating characteristic (ROC) curve analysis. Logistic regression was used to analyze the relative risks (RR) and 95\% confidence intervals for patient status and TRAP assay as continuous variables.

All analyses were performed using the SAS Statistical software (version 9.1, SAS Institute, Cary, NC) and statistical significance for all tests was taken as $\rho<0.05$.

\section{Results}

Telomerase activity of the uterine cervix did not significantly differ as a function of patient age. In fact, TA was $38(0-119)$ in women <35 years, $31(12-95)$ for the 36-45 decade, and 35 (0-76) for the 46-55 decade. A higher, albeit not significantly different value (56; 11-84) was observed in the 6 women $>56$ years of age. Similarly, TA of the uterine cervix did not vary significantly between benign lesions and CIN. In particular, median TA was 35 AEU for patients with inflammation and/or metaplasia and or acanthosis, 29 AEU for CIN1, 36 AEU for CIN2 and 27.5 AEU for CIN3 (Table 1).

Conversely, analysis in relation to clinical outcome showed an almost twofold significant increase $(\rho=0.007)$ in the median AEU value (57 AEU) of TA in the 6 patients whose disease evolved compared to the AEU median value of 31 in disease-free individuals. Of the 6 patients who relapsed, 5 had initial inflammation with acanthosis and/or metaplasia and 1 had a CIN2 
Table 1

TRAP (AEUs) of uterine cervix and 5-year clinical outcome

\begin{tabular}{|c|c|c|c|c|c|c|c|}
\hline & \multicolumn{2}{|r|}{ Overall series } & \multicolumn{2}{|c|}{ Disease-free patients } & \multicolumn{3}{|c|}{ Patients with CIN evolution } \\
\hline & $n$ & $\overline{\text { AEUs Median (range) }}$ & $\bar{n}$ & AEUs Median (range) & $n$ & AEUs Median (range) & $\rho$ \\
\hline Overall series & 62 & $35(0-119)$ & 56 & $31(0-95)$ & 6 & $57(45-119)$ & 0.007 \\
\hline Healthy* & 44 & $36(0-119)$ & 39 & $34(0-95)$ & 5 & $65(45-119)$ & 0.017 \\
\hline Cin 1 & 7 & $29(13-62)$ & 7 & $29(13-62)$ & - & - & \\
\hline Cin 2 & 7 & $36(0-53)$ & 6 & $36(0-53)$ & 1 & 47 & \\
\hline Cin 3 & 4 & $27.5(21-65)$ & 4 & $27.5(21-65)$ & - & - & \\
\hline
\end{tabular}

*Inflammation and/or metaplasia and/or acanthosis.

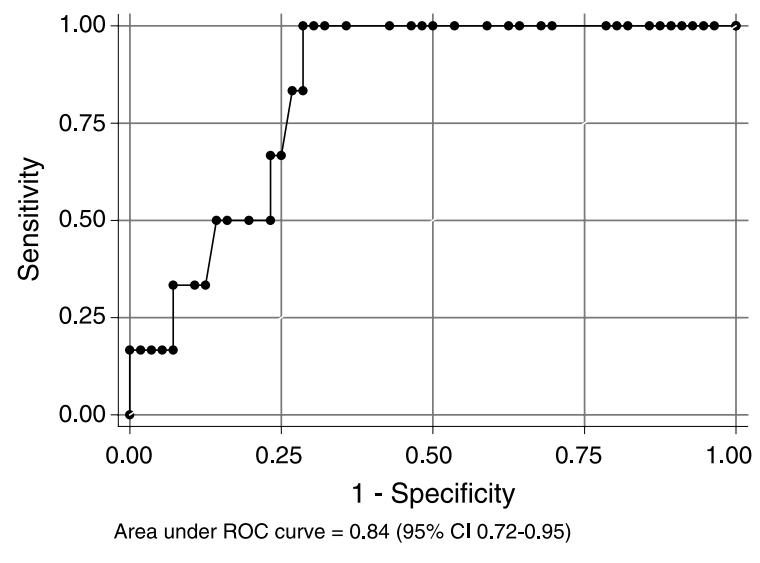

Fig. 3. ROC curve of TRAP assay accuracy.

lesion. In particular, median TA was 65 AEU (45-119) in patients who presented CIN evolution, 34 AEU (0-95) in patients with benign lesions who remained disease-free, 47 AEU in the only CIN2 patient who relapsed and $36 \mathrm{AEU}$ in those with non-recurring CIN2. Any correlation between TA and recurrence was independent of having performed conization treatment (data not shown).

The area under the curve (AUC) for TRAP assay was 0.84 (95\% CI 0.72-0.95) (Fig. 3). Logistic regression showed an unadjusted RR of 1.05 (95\% CI 1.01-1.09, $\rho=0.012$ ), indicating that for a one-unit increase in TRAP we would expect to see a $5 \%$ increase in the odds of being a patient with disease evolution. $100 \%$ sensitivity and $71.5 \%$ specificity were obtained at a cut-off of 45 AEU.

\section{Discussion}

The accurate identification of individuals with benign cervical lesions who are at risk of developing dysplastic lesions or of patients whose dysplastic lesions are destined to recur would greatly facilitate patient monitoring. In the present study we highlighted the ability of TA to identify patients with CIN evolution or recurrence. Telomerase would, in fact, appear to be able to identify patients with benign disease, inflammation and/or metaplasia and/or acanthosis who will relapse with CIN within 5 years. These data suggest that telomerase activity may have a predictive role in the evolution or recurrence of disease and could thus be used in a clinical setting as a marker of high risk of relapse. The relationship between TA and recurrence was independent of having performed or not conisation treatment (data not shown). Prognostic relevance was not observed when telomerase was determined by an immunohistochemical approach [1]. A number of hypotheses can be put forward to explain the presence of hTERT protein in cells with no telomerase reactivation, the most likely being the existence of post-transcriptional and post-translational modes of telomerase regulation, e.g. reversible hTERT protein phosphorylation, the influence of inhibitory factors that can block hTERT protein functions. or the existence of inactive splice variants [1].

Evaluation of a larger case series is ongoing to confirm these results. We are also conducting a study aimed at analyzing the correlation between HPV status and TA activity and at defining the prognostic relevance of TA in patients with HPV positive or negative status.

\section{Acknowledgments}

The authors thank Gráinne Tierney for editing the manuscript.

\section{Conflict of interest}

There are no conflicts of interest to declare. 


\section{References}

[1] S. Bravaccini, M.A. Sanchini, A. Amadori, L. Medri, L. Saragoni, D. Calistri, F. Monti, A. Volpi and D. Amadori, Potential of telomerase expression and activity in cervical specimens as a diagnostic tool, J Clin Pathol 58 (2005), 911-914.

[2] E.A. Jarboe, K.L. Liaw, L.C. Thompson, D.E. Heinz, P.L. Baker, J.A. McGregor, T. Dunn, J.E. Woods and K.R. Shroyer, Analysis of telomerase as a diagnostic biomarker of cervical dysplasia and carcinoma, Oncogene 21 (2002), 664-673.

[3] N.W. Kim, M.A. Piatyszek, K.R. Prowse, C.B. Harley, M.D. West, P.L. Ho, G.M. Coviello, W.E. Wright, S.L. Weinrich and J. Shay, Specific association of human telomerase activity with immortal cells and cancer, Science 266 (1994), 2011-2015.

[4] R.J. Kurman, G.D. Malkasian Jr, A. Sedlis and D. Solomon, From Papanicolaou to Bethesda: The rationale for a new cervical cytologic classification, Obstet Gynecol 77 (1991), 779-782.

[5] M. Porika, R. Tippani, A. Mohammad, S.R. Bollam, S.D. Panuganti and S. Abbagani, Evaluation of serum human telomerase reverse transcriptase as a novel marker for cervical cancer, Int J Biol Markers 26 (2011), 22-26.

[6] M.A. Sanchini, S. Bravaccini, L. Medri, R. Gunelli, O. Nanni, F. Monti, P.C. Baccarani, A. Ravaioli, E. Bercovich, D. Amadori and D. Calistri, Urine telomerase: An important marker in the diagnosis of bladder cancer, Neoplasia 6 (2004), 234-239.

[7] J.W. Shay, Y. Zou, E. Hiyama and W.E. Wright, Telomerase and cancer, Hum Mol Genet 10 (2001), 677-685.

[8] M. Takakura, S. Kyo, T. Kanaya, M. Tanaka and M. Inoue, Expression of human telomerase subunits and correlation with telomerase activity in cervical cancer, Cancer Res 58 (1998), 1558-1561.

[9] G.B. Wisman, S. De Jong, G.J. Meersma, M.N. Helder, H. Hollema, E.G. de Vries, W.N. Keith and A.G. van der Zee, Telomerase in (pre)neoplastic cervical disease, Hum Pathol 31 (2000), 1304-1312.

[10] W.E. Wright, J.W. Shay and M.A. Piatyszek, Modifications of a telomeric repeat amplification protocol (TRAP) result in increased reliability, linearity and sensitivity, Nucleic Acids Res 23 (1995), 3794-3795. 


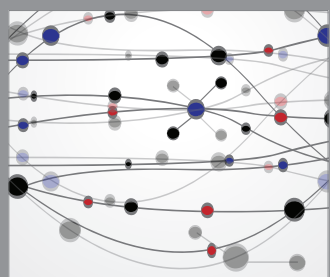

The Scientific World Journal
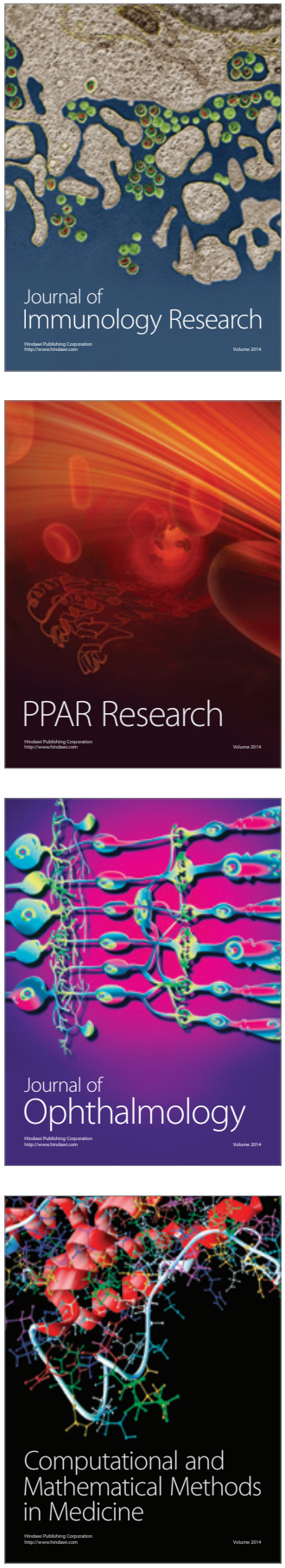

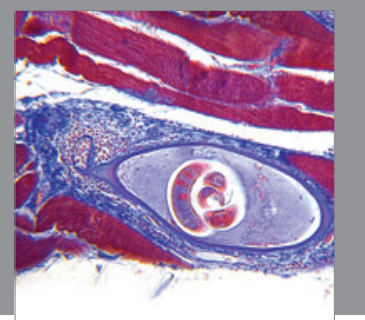

Gastroenterology

Research and Practice
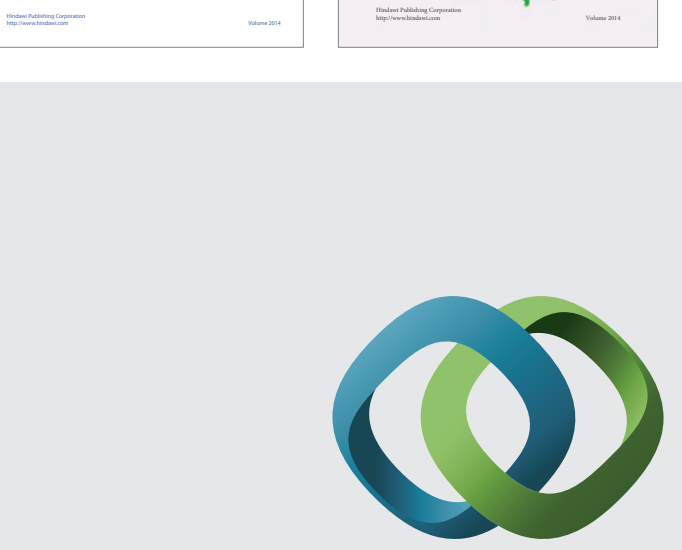

\section{Hindawi}

Submit your manuscripts at

http://www.hindawi.com
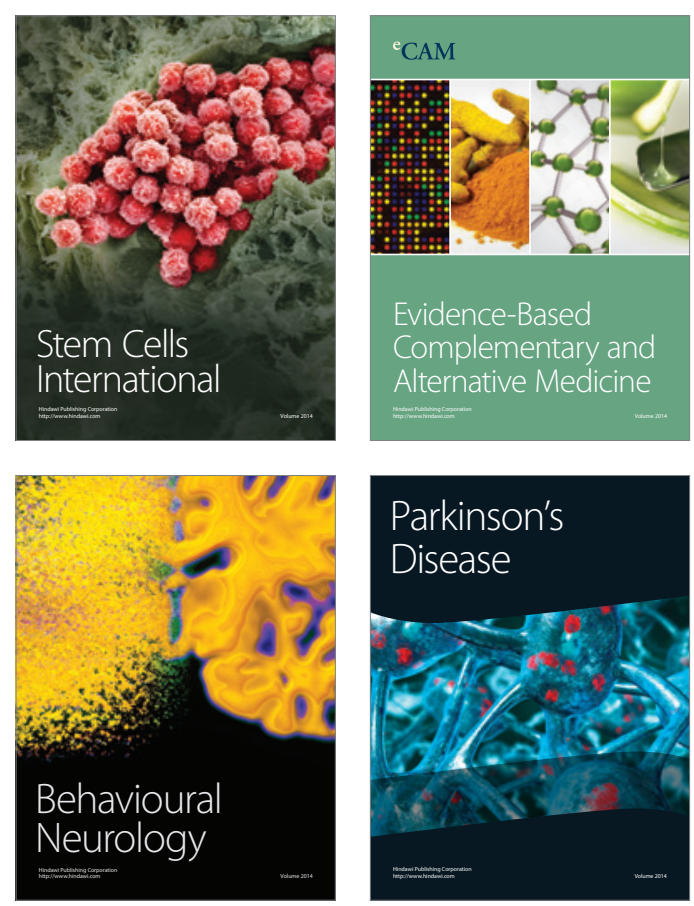

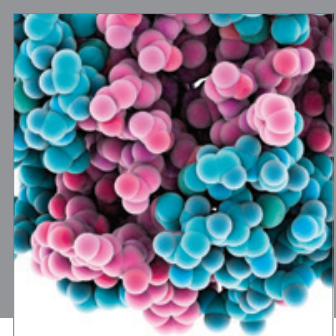

Journal of
Diabetes Research

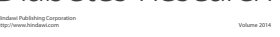

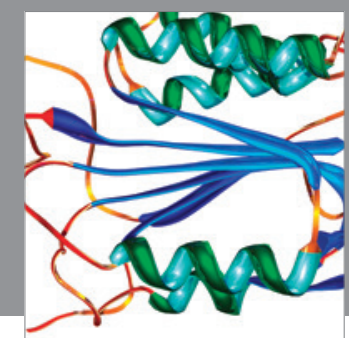

Disease Markers
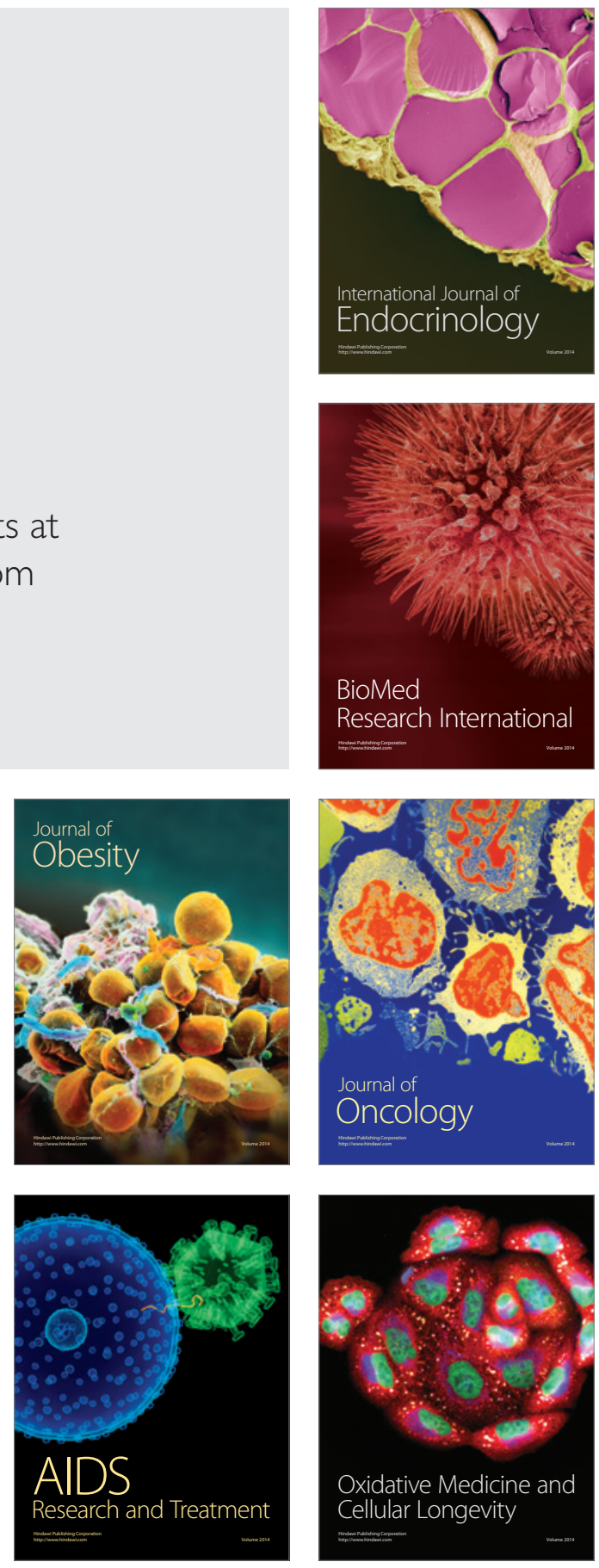